Citation: F. Zannoni (2020) La didattica universitaria a distanza durante e dopo la pandemia: impatto e prospettive di una misura emergenziale. Media Education 11(2): 75-84. doi: 10.36253/ me-8979

Received: June, 2020

Accepted: September, 2020

Published: December, 2020

Copyright: (c) 2020 F. Zannoni. This is an open access, peer-reviewed article published by Firenze University Press (http://www.fupress.com/me) and distributed under the terms of the Creative Commons Attribution License, which permits unrestricted use, distribution, and reproduction in any medium, provided the original author and source are credited.

Data Availability Statement: All relevant data are within the paper and its Supporting Information files.

Competing Interests: The Author(s) declare(s) no conflict of interest.

\section{La didattica universitaria a distanza durante e dopo la pandemia: impatto e prospettive di una misura emergenziale}

\author{
University distance teaching during and after the pandemic: \\ impact and perspectives of an emergency measure
}

FEDERICo ZANNONI

Università degli Studi di Bologna

E-mail: federico.zannoni3@unibo.it

\begin{abstract}
Participation in online lessons, delivered on virtual platforms to replace traditional face-to-face activities, took on a significant role in the daily life of numerous university students during the quarantine imposed on a national scale in response to the pandemic emergency. A qualitative survey conducted on a sample of students enrolled in the School of Medicine and Surgery of the University of Bologna allowed to analyse the impact and the factors of positivity and negativity of online lessons, providing elements to reflect on possible developments, when this method will cease to be bound to the emergency, to become an opportunity to be integrated with traditional face-to-face learning.
\end{abstract}

Keywords: university teaching, distance teaching, quarantine, sanitary emergency, post-pandemic teaching.

Riassunto. La partecipazione alle lezioni online, erogate su piattaforme virtuali in sostituzione alle tradizionali attività didattiche in presenza, ha assunto un ruolo significativo nella quotidianità di numerosi studenti universitari durante il periodo della quarantena imposta su scala nazionale in risposta all'emergenza pandemica. Un'indagine qualitativa condotta su un campione di studenti iscritti ai corsi afferenti la Scuola di Medicina e Chirurgia dell'Università di Bologna ha consentito di analizzare l'impatto, gli elementi di positività e quelli di negatività delle lezioni online, fornendo spunti per riflettere sui possibili sviluppi, quando tale modalità smetterà di essere vincolante in quanto legata allemergenza, per divenire una opportunità da integrare alle forme tradizionali in presenza.

Parole chiave: didattica universitaria, didattica a distanza, quarantena, emergenza sanitaria, didattica post-pandemica. 


\section{INTRODUZIONE}

La cronologia che ha caratterizzato, a livello mondiale, le fasi del debutto e dell'esplosione della pandemia di Covid-19 ha sancito il ricorso nei diversi paesi, a giorni o settimane di distanza l'uno dall'altro, a provvedimenti non privi di punti di contatto, volti a intervenire sulle medesime sfere della vita comunitaria: tra queste, la gestione dell'istruzione scolastica e universitaria. A due mesi dal riconoscimento del primo paziente con sinto$\mathrm{mi}$, avvenuto in Cina il $1^{\circ}$ dicembre 2019 (Huang et al., 2020), il 30 gennaio 2020 l'Organizzazione Mondiale della Sanità dichiara lo stato di emergenza sanitaria pubblica di interesse internazionale (Lai et al., 2020) e l'11 marzo 2020 comunica ufficialmente la presenza della pandemia di Covid-19 (World Health Organization, 2020).

Il 13 marzo 2020, 61 paesi in ogni parte del mondo annunciano la chiusura di scuole e università e il ricorso a modalità di apprendimento a distanza (UNESCO, 2020). Alla base di queste decisioni vi è la forte contagiosità del virus, trasmissibile soprattutto per via orale, e la possibilità che possa essere diffuso da persone asintomatiche, o blandamente sintomatiche, in modo particolare dai più giovani, più raramente soggetti a manifestazioni acute (Munster et al., 2020). Alla luce di questo quadro virologico e della conseguente necessità di adottare politiche preventive di distanziamento sociale, le scuole e le università sono state ben presto identificate come ambienti particolarmente vulnerabili, ad alto rischio, in quanto frequentati da migliaia di studenti, a stretto contatto reciproco, spesso affollati in aule stipate che, in presenza di asintomatici contagiosi, avrebbero potuto costituire un forte elemento di pericolosità e incertezza.

Simili interventi erano già stati realizzati nel passato, per affrontare altre situazioni di emergenza epidemica o di catastrofe naturale. Nella primavera 2009, nel bel mezzo della grande recessione, il $67 \%$ dei piani elaborati per fronteggiare l'influenza pandemica H1N1 (influenza suina) contemplavano il passaggio dalla didattica in classe $o$ in aula alle modalità online (Allen \& Seaman, 2010); identici provvedimenti sono stati presi in Texas e nella regione del Golfo nell'agosto 2005, quando l'urgano Katrina provocò ingenti danni in 27 colleges americani (Meyer e Wilson, 2011): ne conseguì l'attuazione del cosiddetto Sloan Semester, che impegnò un consorzio di 153 colleges nella creazione di un catalogo di 1300 corsi a distanza.

In questi casi come nell'attuale situazione di pandemia di Covid-19, il passaggio dalla didattica in presenza a quella a distanza costituisce una misura di emergenza atta a consentire l'attuazione del distanziamento sociale, a sua volta finalizzato a scongiurare il pericolo della comparsa di focolai epidemici nelle scuole e nelle università. Vista la gravità dell'emergenza, la scelta della chiusura delle istituzioni educative, attuata su scala nazionale in seguito a direttive ministeriali, in Italia così come in numerosi altri paesi, si caratterizza come una misura straordinaria, mossa da motivazioni di sicurezza e protezione, che nella precisa circostanza divengono prioritarie rispetto a istanze di diverso tipo, ad esempio relative alla qualità dell'insegnamento e alla libera determinazione dei singoli organi collegiali e amministrativi. Il passaggio dalla didattica in presenza all'e-Learning è stato originato, presentato e legittimato come una misura di sicurezza per proteggere la comunità dal pericolo di diffusione del virus, attuato dalle autorità preposte all'istruzione con forte sostegno e coinvolgimento da parte degli esperti in materia di virologia e sanità. In altre parole, abbiamo assistito a un processo di costruzione sociale della rappresentazione delle lezioni scolastiche e universitarie in presenza come fattori di rischio per la sicurezza nazionale, alla cui definizione hanno partecipato i decisori politici, gli scienziati e gli esperti, i mezzi di informazione, la cittadinanza.

Questo tipo di approccio riflette le teorizzazioni a loro tempo elaborate dagli studiosi della Scuola di Copenaghen (Weawer, 1995; Buzan et al., 1998), le cui formulazioni classiche partono dall'assunto di base secondo cui, nelle situazioni di pericolo su grande scala, il successo dei provvedimenti di messa in sicurezza dipende dalla felice interazione tra le scelte politiche e amministrative e l'assenso della popolazione a tali azioni e decisioni. Nelle situazioni emergenziali, i soggetti politici assumono poteri e autonomie decisionali straordinari, superiori a quelli legittimabili in condizioni di normalità, che devono tuttavia rimanere circoscritti alla durata dell'emergenza securitaria ed essere seguiti da un repentino processo di allentamento e de-securitizzazione che possa riportare all'equilibrio precedente il pericolo.

L'attuale ribalta a cui è pervenuta la didattica a distanza è stata possibile anche e soprattutto grazie alla ormai lunga storia che si porta dietro, le cui origini possono essere rintracciate nelle prime decadi del Novecento, quando comparvero circoscritte manifestazioni (Birnbaum, 2001), divenute più frequenti a partire dal secondo dopoguerra, soprattutto negli Stati Uniti, nelle forme di contributi didattici che si avvalevano di comunicazioni telefoniche, testi scritti, audiocassette e videocassette spedite per posta, programmi radiofonici e televisivi, per raggiungere persone che vivevano in aree rurali o poco urbanizzate (Matthews, 1999), così come militari in missione e veterani di guerra. La più recente diffusione di personal computer e della rete Internet, cui è corrisposto l'irrompere delle prime generazioni di nativi 
digitali (Ranieri, 2011), divenuti ormai giovani adulti, ha rivoluzionato il mondo dell'e-Learning, aprendo la strada al suo ricorso presso le università pubbliche e private.

Terry Anderson (2011) definisce la didattica online come una tra le tante possibilità di insegnamento $\mathrm{e}$ apprendimento attualmente esistenti, avente come peculiarità la possibilità di offrire un accesso all'esperienza educativa più flessibile per quanto riguarda gli spazi e i tempi, dal momento che non implica la compresenza in un determinato luogo di docente e discenti e contempla la fruizione asincrona; tale caratteristica le consente di essere potenzialmente accessibile a un numero incredibilmente elevato di utenti, raggiungendo soggetti ai quali la frequenza a forme in presenza sarebbe preclusa, per motivi personali, lavorativi, economici, geografici (Traxler, 2018). Sempre secondo Anderson (2011), un ulteriore aspetto che la contraddistingue è il superamento del testo scritto come unico ambiente di apprendimento lineare e la proposta di un approccio multimediale, che sappia «abbandonare la logica sequenziale del manuale» (Persico e Midoro, 2013, p. 10) per procedere in modo ipertestuale e reticolare, favorendo l'interazione tra i saperi e una concezione della conoscenza come perpetua costruzione e ridefinizione attuata dal soggetto in modo attivo, opportunamente sollecitato da stimoli provenienti sia dalla cosiddetta educazione formale, sia da quella informale e non formale (Bonaiuti, 2006). L'efficacia dei percorsi di e-Learning poggia in buona parte sul grado di autonomia che il fruitore sa esercitare e padroneggiare (Firat, 2016; Jacobs, Renandya \& Power, 2016), in quanto egli stesso, libero da controlli e condizionamenti, è responsabile del successo del proprio processo di apprendimento (Campione, 2015; Margiotta, 1999).

Passando in rassegna gli studi più rilevanti, Manijeh Sadeghi (2019) individua i vantaggi e gli svantaggi dell'apprendimento a distanza. Tra i primi, oltre alla già citata possibilità di essere esperito in qualsiasi posto e, se organizzato in forma asincrona, in qualunque momento (Nagrale, 2013), menziona il risparmio economico, riconducibile non solo a tasse di iscrizione più basse, ma anche all'evitamento dei costi per gli spostamenti e gli eventuali cambi di domicilio; la pratica del pendolarismo, inoltre, comporterebbe un significativo dispendio non solo di denaro, ma anche di tempo ed energie. La flessibilità, talvolta prevista anche nella scelta dei moduli d'apprendimento, e la possibilità di non essere studenti a tempo pieno, ma continuare a esercitare la propria professione, costituiscono ulteriori punti di forza del ricorso all'e-learning.

Di contro, non mancano gli svantaggi. Innanzitutto, l'assenza di interazioni faccia a faccia e la non prossimità fisica ai compagni possono favorire la distrazione dalla lezione, a meno che l'individuo non sia estremamente motivato e in grado di autodisciplinarsi; inoltre, in caso di dubbi o necessità di chiarimenti, il confronto con il docente potrebbe essere meno immediato ed esaustivo. L'eccessiva dipendenza dalle tecnologie, oltre a limitare i processi comunicativi, implicherebbe la non infrequente eventualità di incorrere in problemi tecnici, di funzionamento $o$ di disponibilità di connessione e rete, così come la mancata compresenza di studenti e professori in un'unica aula istillerebbe vissuti di isolamento e sofferenza riconducibili al bisogno insoddisfatto di interazione sociale, fisica e verbale (Dyrud, 2000), a cui gli scambi tramite chat, email e videoconferenze non possono sopperire appieno. Le difficoltà e le angosce esperite dagli studenti non sono completamente comprensibili attraverso le sole interazioni online e spie di depressione riconducibili all'isolamento potrebbero non essere riconosciute per tempo (Hara \& Kling, 2000).

Alla luce dei numerosi limiti che la didattica online a livello universitario mantiene, se assunta come unica modalità, ma anche dell'evidenza di come da più parti siano in aumento le spinte, già presenti prima dell'attuale pandemia, ma ora più legittimate e insistenti, agite da commentatori politici e istituzioni private di apprendimento a distanza in nome rispettivamente dell'austerità e del profitto, affinché essa possa prendere definitivamente e strutturalmente il posto di quella tradizionale in presenza (Blumenstyk, 2020), Michael Murphy (2020) ribadisce con forza la necessità di non cadere nella facile tentazione di normalizzare strategie didattiche ed educative attuate come risposta all'emergenza pandemica, e quindi per loro natura provvisorie.

\section{METODO}

Attraverso una e-mail inviata al corpo docente e agli studenti domenica 23 febbraio 2020, il Rettore dell'Università di Bologna, in considerazione dell'evoluzione del fenomeno epidemico legato al diffondersi dell'infezione coronavirus Covid-19, in accordo con il Ministero dell'Università e della Ricerca, con la Regione EmiliaRomagna e le altre Università della Regione, comunica la sospensione dell'attività didattica in presenza in tutte le sedi dell'Ateneo; a distanza di una settimana, una successiva missiva avverte dell'inizio, già a partire da lunedì 2 marzo 2020, dell'erogazione di attività formative nella modalità della didattica online, attraverso la piattaforma Microsoft Teams, con orario invariato rispetto al periodo precedente l'interruzione.

Tra gli studenti che si trovano ad affrontare questo repentino cambiamento, vi sono gli iscritti ai corsi di 
laurea offerti dalla Scuola di Medicina e Chirurgia. Dal principio della propria carriera universitaria abituati a frequentare lezioni in presenza, attività laboratoriali e tirocini sul campo, ma assai di rado esperienze di apprendimento online, questi studenti e queste studentesse si sono trovati di soppiatto a fare i conti con una realtà tutta nuova, che si inserisce in una situazione di diffusione del contagio e conseguente inasprimento dei provvedimenti di distanziamento sociale che, settimana dopo settimana, si fa sempre più netta e radicale, foriera di stravolgimenti e preoccupazioni.

La presente ricerca intende analizzare l'impatto che l'introduzione della didattica online ha avuto sulle modalità di apprendimento e sulle rappresentazioni del proprio ruolo di studenti e studentesse. In modo particolare, si è inteso richiedere una meta-riflessione sugli elementi positivi e negativi non solo della didattica online, ma specularmente anche di quella tradizionale in presenza, nonché su come l'impegno nel proprio ruolo di studenti e studentesse, seppure con modalità inedite, abbia potuto contribuire a permettere di affrontare il disorientamento e gli effetti critici, a livello psicologico, di organizzazione della routine quotidiana e di percezione del futuro, prodotti dalla quarantena.

Il fine ultimo è quello di distinguere gli elementi riconducibili alla situazione emergenziale e quelli che invece viene ritenuto si possano continuare a proporre anche una volta terminata la pandemia, perché rivelatisi funzionali a sostituire, o per lo meno ad affiancare e integrare, le strategie e le modalità tradizionali. Tali finalità si possono tradurre nelle seguenti domande di ricerca:

- Durante i mesi della quarantena, che importanza ha avuto il poter comunque continuare a svolgere il proprio ruolo di studente o studentessa, pur in solitudine tra le mura di casa?

- Quali sono stati gli elementi di positività e quali quelli di negatività insiti nella didattica a distanza?

- Una volta ristabilitasi la possibilità di un ritorno a una didattica in presenza, sarà ancora preferibile tale forma oppure l'esperienza della didattica a distanza potrà essere estesa, in modo parziale $o$ totale, anche oltre il periodo emergenziale?

Tali domande si sono declinate nei seguenti 8 questi, somministrati attraverso interviste:

1) Quali sono gli aspetti positivi del seguire le lezioni online?

2) Quali sono gli aspetti negativi?

3) Ritieni che le lezioni online consentano ai docenti di migliorare l'efficacia della propria didattica? Come e perché?

4) Ritieni che le lezioni online limitino e penalizzino le possibilità didattiche dei docenti? Come e perché?
5) Nel complesso, sei soddisfatto/a? Preferisci questa modalità o la forma tradizionale di lezione in presenza?

6) Quale dispositivo usi?

7) Quante ore giornaliere/settimanali passi ad assistere alle lezioni online? Ritieni tale monte ore adeguato, eccessivo o troppo esiguo? Perché?

8) In questo periodo di distanziamento sociale da trascorrere per lo più presso le proprie case, quale importanza/ulteriore funzione rivestono le lezioni online?

Hanno risposto 100 studenti e studentesse (35 maschi e 65 femmine), frequentanti i corsi di laurea di Medicina e Chirurgia, Fisioterapia e Tecniche di prevenzione nell'ambiente e nei luoghi di lavoro, in quel periodo impegnati nel seguire lezioni online.

Le interviste sono state effettuate a tre settimane dall'inizio dei corsi a distanza, nella fase più acuta dall'epidemia, momento in cui le fatiche dell'isolamento domestico cominciavano a farsi sentire con prepotenza e un primo bilancio sull'approccio alla didattica online poteva essere fatto. Questa ricerca si propone finalità qualitative, dal momento che, rifacendosi all'approccio della thematic analysis, particolarmente adatto per la sua flessibilità (Braun e Clarke, 2006), ha inteso individuare nelle risposte ottenute alcuni nuclei tematici, rispetto ai quali, nel successivo paragrafo, viene restituita una prima analisi, strettamente legata al contesto emergenziale.

\section{RISULTATI}

\subsection{La situazione emotiva}

Il punto di partenza più opportuno sui cui ancorare la restituzione degli esiti della ricerca costituisce nel riportare le risposte che più di altre riescono a delineare il contesto emotivo ed esperienziale su cui il ricorso alla patica delle lezioni online è intervenuto.

Per la quasi totalità del campione (96\%), le lezioni online durante i giorni della quarantena hanno costituito delle sorte di assi su cui impostare una nuova routine quotidiana, momenti fissi che hanno permesso non solo di tenersi occupati e impegnati, ma anche di distrarsi dai pensieri più cupi, ansiogeni e dolorosi riferibili all'epidemia. Hanno inoltre consentito di trovare motivazioni e risorse per contrapporsi all'abbandono, alla pigrizia, all'apatia e al passare il tempo senza fare nulla di interessante, bensì giocherellando, cucinando o guardando la televisione. Nelle interminabili giornate trascorse in casa, hanno contribuito a tenere la mente occupata in modo proficuo, allontanano le tentazioni di uscire nonostante i divieti e i fattori di rischio. 
Le lezioni online mi hanno permesso di occupare la giornata e ritrovare la routine che si era persa. Mi fa piacere riuscire a mantenere in questo modo contatto con gli altri e soprattutto con le persone con le quali ho maggiormente legato, senza interrompere per questa emergenza i rapporti creatisi.

Sicuramente consentono di mantenere l'idea di normalità e consentono di interfacciarsi con altre persone oltre ai propri famigliari con cui si è in casa tutto il giorno.

Hanno la funzione di non farci perdere le nostre abitudini e le nostre certezze riguardanti il futuro. Nel limite del possibile, ci consentono di mantenere una routine, di non perdere il senso del tempo, di essere attivi.

Le lezioni online hanno permesso di limitare la sensazione di cesura con la vita che si conduceva prima della quarantena, facilitando il mantenimento di un contatto con la propria realtà di studenti di Medicina e con una idea di normalità in cui è ancora possibile conservare la mente attiva, ritenere utile e sensato il modo in cui si sta passando il tempo, non perdere le abitudini e le certezze riguardanti il futuro, non smarrire il senso del tempo e, non da ultimo, colmare le distanze fisiche e sociali continuando a interagire con i compagni di corso. Hanno offerto momenti di condivisione, socialità intellettuale e interazione, contrapponendo alla solitudine il farsi compagnia percependo le reciproche presenze e dialogando tramite chat e video-interventi. Soltanto poche persone ( $4 \%$ del campione) hanno negato questi ulteriori significati, dichiarandosi scarsamente coinvolte nella vita universitaria, a riprova di come l'irrompere delle lezioni online durante l'isolamento si sia rivelato anche un'utile cartina tornasole per stimolare una riflessione personale su quanto soddisfacente, sino a quel momento, sia stata l'esperienza universitaria, uno spartiacque da cui trarre ulteriori motivazioni per non interrompere il percorso di studi, non rimanere indietro e preparare gli esami, restando focalizzati sugli obiettivi personali, accademici e professionali.

\subsection{Le potenzialità logistiche}

Proiettandosi oltre le condizioni psicologiche e sociali legate al momento emergenziale, non mancano gli elementi di positività in quanto tale individuati nella didattica online da studenti e studentesse, primi tra tutti la possibilità di limitare gli spostamenti e non essere soggetti ai disagi del pendolarismo. Gli spostamenti con il treno e altri mezzi pubblici occuperebbero molto tempo, sottraendolo allo studio e agli impegni famigliari e personali, e provocherebbero sovente ritardi, talvol- ta grandi al punto tale da fare perdere intere lezioni. La vita del pendolare sarebbe faticosa, in quanto implicherebbe l'alzarsi molto presto la mattina e l'arrivare a casa stanchi la sera, senza considerare i costi di abbonamenti e biglietti, notevoli se rapportati a certe disponibilità economiche, ma mai quanto gli affitti per coloro che decidono di trasferirsi, a maggior ragione viste le effettive condizioni delle abitazioni. La consapevolezza di non dover occupare lunghe ore negli spostamenti permetterebbe ai pendolari di concentrare le energie e l'attenzione nelle ore di lezione online, nella rasserenante consapevolezza di avere successivamente tempo a disposizione per dedicarsi ad altro.

È più comodo seguire le lezioni da casa senza doversi spostare, e quando tutto funziona correttamente si riesce a seguire senza interruzioni e brusio di fondo.

La logistica! Avendo lezioni sparse in vari posti, si recupera un sacco di tempo e per i pendolari non è necessario spostarsi e anche chi ha altre difficoltà organizzative può essere estremamente facilitato. Oltre al fatto che non è necessario stiparsi in anfiteatri non più a norma.

La fruizione domestica delle lezioni permetterebbe di stare seduti con più agio sul proprio divano o alla scrivania, avendo ogni cosa a portata di mano, rimanere in pigiama o comunque in tenuta casalinga, senza l'ulteriore impegno di vestirsi e acconciarsi in modo adeguato; consentirebbe di fare colazione con i coinquilini o i famigliari guardando il professore mentre spiega, andare in bagno in qualsiasi momento, dormire di più, finanche occuparsi delle faccende domestiche e cucinare, o comunque fare altro. Ben altra cosa sarebbero i ricordi di aule stipate oltre le norme di sicurezza e di spostamenti frettolosi da una stanza all'altra nei palazzi universitari, con inevitabili ritardi negli orari di inizio e conseguenti lezioni affrettate e programmi non portati a termine. La puntualità e il rispetto degli orari, più garantiti nelle lezioni online, aumenterebbero efficacia, concentrazione e profitto negli studi.

Per alcuni, le modalità didattiche online permetterebbero di seguire meglio la lezione ed essere soggetti a meno distrazioni. Nelle aule universitarie le possibilità di distrazione sarebbero infatti moltissime: telefoni che vibrano, chiacchiere tra compagni, confusione, brusio, rumori di sottofondo, persone che entrano a lezione iniziata, altre che non seguono, interruzioni, perdite di tempo. Tali occasioni per distrarsi comprometterebbero le soglie di attenzione degli studenti e renderebbero più difficoltosa per i professori la conduzione della lezione. L'ambiente online annullerebbe questi rischi, eludendo il problema della gestione della disciplina e del silenzio 
in aula. Sarebbe quindi possibile sentire più nitidamente la voce del docente, mantenere la concentrazione per più tempo e prendere meglio gli appunti, non essendo distratti. Per altri, al contrario, l'essere da soli in una stanza, senza sottostare ad alcuna forma di controllo, impegnati in attività che implicherebbero una partecipazione meno attiva, in assenza della presenza fisica del professore, e quindi nell'impossibilità di cogliere la sua mimica e il suo linguaggio non verbale, costituirebbero elementi che faciliterebbero distrazioni e la difficoltà nel mantenere la concentrazione.

\subsection{I materiali e i metodi d'insegnamento e apprendimento}

Dal momento che il materiale viene condiviso dal docente direttamente sulla piattaforma, si vedrebbero meglio le slides (perché più vicine, sullo schermo), le registrazioni audio della lezione sarebbero di qualità migliore e si avrebbe tutto il materiale a disposizione sul personal computer. Il superamento di talune limitazioni fisiche consentirebbe di non perdere tempo prezioso; inoltre, la piattaforma Microsoft Teams sarebbe piuttosto intuitiva e di semplice utilizzo. Ciononostante, imprevisti di tipo tecnico non sarebbero rari: problemi di connessione e nei sistemi audio e video, interruzioni improvvise, errori nella trasmissione dei link di accesso alle attività, files non leggibili da alcuni computer, calendario online non aggiornato, procedure difettose per registrare le presenze e, non da ultima, inesperienza nell'uso del sistema, soprattutto da parte dei docenti, dal momento che alcuni di loro si destreggerebbero male nell'utilizzo delle piattaforme, compiendo errori anche grossolani e non sfruttandone appieno le potenzialità.

Non avere a disposizione una lavagna allunga i tempi, così come problemi di connessione e poca confidenza con la tecnologia possono ridurre la qualità dei materiali didattici che potrebbero invece essere proiettati o mostrati a lezione.

Le slide e l'audio sul computer aiutano, ma vedere una persona dal vivo che spiega è molto bello ed entusiasmante.

La modalità online renderebbe più difficili, in certi casi impossibili, le attività di tipo pratico, l'illustrazione di casi clinici e le esercitazioni laboratoriali e di gruppo, e poco calzerebbe a quelle discipline che, come psicologia, pedagogia e statistica, necessitano di maggiore confronto e interazione. Alcuni docenti sarebbero più predisposti rispetto ad altri, ma, in ogni caso, un buon insegnante sarebbe comunque tenuto ad assicurare una offerta didattica di qualità, a prescindere dal mezzo utilizzato, adattando a esso i contenuti. Questo non sempre accade: quando si riducono a letture di slides, le lezioni sarebbero noiose; inoltre, molto spesso i professori sarebbero portati a parlare più velocemente rispetto a come farebbero in aula e a fare meno pause, anche tra una lezione e l'altra, risultando più difficili da seguire, così come la mancanza della lavagna ridurrebbe l'intuitività e l'immediatezza delle spiegazioni.

\subsection{Mutamenti nell'interazione}

Venendo meno la presenza fisica delle persone in uno stesso luogo, cambiano le forme della relazione. $\mathrm{Ne}$ risentirebbero la qualità e la quantità della partecipazione e delle interazioni tra studenti e docenti e tra studenti e studenti, dal momento che per alcuni risulterebbe più difficile partecipare attivamente, considerando la comunicazione tramite computer "triste", alienante, noiosa, più difficile, imbarazzante, limitata e limitante il dialogo e il confronto. Private del rapporto umano col docente, che nelle lezioni in presenza lascia il segno al di là dei soli contenuti, le lezioni diverrebbero aride e poco attrattive; inoltre, non potendo vedere le facce di professori e compagni nemmeno tramite webcam, dal momento che devono rimanere spente per evitare di sovraccaricare il sistema, si perderebbero le componenti comunicative della mimica facciale e del linguaggio non verbale. In definitiva, verrebbe meno, o comunque sarebbe fortemente compromessa, la dimensione della socialità, per alcuni parimenti importante rispetto a quella dell'apprendimento.

C'è meno interazione tra compagni e con i professori, è un po' triste stare chiusi in camera da soli senza poter parlare di persona. A volte la connessione internet si blocca, ci sono normalissimi problemi tecnici che possono rallentare o creare problemi nella comunicazione tra studente e professore.

In controtendenza rispetto a quanto detto in riferimento a un buon numero di rispondenti, alcuni studenti e studentesse ammettono di sentirsi inibiti nel fare interventi e domande al professore durante le lezioni in presenza: per timidezza, per disagio a parlare davanti a molte persone, per vergogna, per paura di interrompere o di chiedere cose fuori luogo. Viceversa, le piattaforme online permetterebbero di interagire con più frequenza ed efficacia, eliminando le inibizioni e i blocchi di natura emotiva, anche grazie all'utilizzo della chat in sostituzione all'intervento orale. Grazie alla chat e all'impersonalità del mezzo tecnologico, che annullerebbero o attenuerebbero le inibizioni che si hanno in aula, per alcuni sarebbero aumentate le domande e le richieste di chia- 
rimento, consentendo così ai docenti di rendersi meglio conto di quanto gli studenti abbiano davvero appreso e compreso. Su quest'ultimo punto dissentono coloro che ritengono che durante le lezioni online il docente non avrebbe la possibilità di rendersi conto se gli studenti abbiano effettivamente capito gli argomenti spiegati, di quanti stiano veramente seguendo e di quanti siano interessati, di quando sia il momento per proporre una pausa, o quando sia necessario ripetere un concetto. Non vedendo i volti degli alunni, il professore non può interpretare le loro reazioni e tenderebbe a procedere troppo veloce nella spiegazione, incontrando maggiori difficoltà nel sollecitare attenzione, interesse, comunicazione $\mathrm{e}$ partecipazione.

I prof, non vedendo le facce degli studenti, vanno più veloci; inoltre alcuni professori non fanno la pausa necessaria tra un'ora e un'altra, motivo per il quale dopo poco lo studente chiude l'applicazione perché non riesce più a seguire.

Gli studenti e le studentesse riconoscono che il passaggio alle lezioni online ha comportato un cambiamento nel modo di fare didattica da parte dei professori. Messi alla prova con modalità ai più inedite, per alcuni aspetti più difficili, viene loro riconosciuto di essersi dovuti mettere in discussione e aver dovuto modificare o cambiare strategie e metodi che magari erano soliti utilizzare da anni. Ora, per poter sopperire all'assenza del contatto diretto, i professori sarebbero costretti a rimanere più concentrati sulla lezione e a impegnarsi di più nella spiegazione, perseguendo una maggiore attenzione alla chiarezza nell'esposizione e alla linearità, soffermandosi di più sugli argomenti, approfondendo, migliorando la qualità e l'esaustività delle slides e del materiale didattico proposto, cercando di essere più interattivi e diretti, divagando meno, anche in ragione di una scansione oraria piuttosto intensa.

\subsection{Il carico giornaliero}

Coloro che mediamente sono stati impegnati nel seguire le lezioni online tra le 4 e le 6 ore al giorno ritengono tale carico adeguato, inferiore rispetto alla circostanza in cui il medesimo monte ore fosse stato esperito in presenza, in quanto quel caso avrebbe comportato l'aggiunta del tempo necessario per gli spostamenti coi mezzi pubblici, le pause e le ore buche tra una lezione e l'altra. Al contrario, per coloro che sono stati connessi per $7 / 8$ ore al giorno, sovente con poche pause, si è rivelato un carico eccessivo, che ha lasciato poco tempo per studiare e ha reso difficile mantenere l'attenzione, soprattutto al pomeriggio, senza considerare la percezione di stare sforzando troppo gli occhi e di rimanere fermi in posizioni poco salutari; un dato di fatto è l'ammissione che quasi nessuno abbia seguito tutte le lezioni, ma tra compagni si è provveduto a scambi e condivisioni di appunti.

\subsection{Il grado complessivo di soddisfazione}

Nel complesso, le lezioni online sono considerate una buona soluzione, probabilmente l'unica possibile, per affrontare il momento dell'emergenza senza interrompere le attività didattiche. Alcuni studenti e studentesse le preferiscono a quelle in presenza anche a prescindere dall'emergenza, considerandole più efficaci soprattutto in relazione ai loro tratti caratteriali e di personalità e alle esigenze personali; consentirebbero di prendere meglio gli appunti, cosa più difficile all'università a causa delle infrastrutture non adeguate, nonché un risparmio economico e l'eliminazione dei disagi legati al pendolarismo. Qualora venisse introdotta anche la modalità asincrona, la possibilità di fruire di videolezioni registrate, l'indice di gradimento sarebbe ancora maggiore.

Nel complesso sono molto soddisfatta e ritengo che questa forma di lezione sia molto più congeniale alle mie esigenze personali.

Al contrario, altri studenti e studentesse auspicano un ritorno alle lezioni in presenza, preferibili in quanto si presterebbero a minori distrazioni, favorirebbero $\mathrm{i}$ rapporti sociali e il contatto umano, sarebbero più complete, formative e di qualità.

Sicuramente è un ottimo lavoro considerando il tempo in cui è stato realizzato e la lacuna che riesce a colmare durante quest'emergenza, ma la forma tradizionale di lezione ha sicuramente un "in più" non indifferente, anche dal punto di vista dell'efficacia del contenuto trasmesso.

Infine, vi sono coloro i quali riscontrano aspetti positivi e altri negativi sia nella modalità online, sia in quella in presenza, e auspicano, per il futuro, l'introduzione di una erogazione mista, che contempli entrambe, in modo da facilitare i pendolari e valorizzare le diverse discipline, affrontandole con più strumenti e strategie.

Per alcuni aspetti preferisco le lezioni in modalità online poiché consentono di gestire meglio il tempo essendo a casa, ma per alcuni insegnamenti preferisco comunque le lezioni in presenza e avere un maggiore dialogo con le persone che mi circondano. 
Nel complesso sono soddisfatta, penso che alcuni corsi, ad esempio quelli elettivi, si potrebbero tenere normalmente in questa modalità per il futuro, dato anche che spesso sono la sera, per cui disagevoli per chi è pendolare. Per il resto delle lezioni preferisco comunque in presenza.

\section{DISCUSSIONE}

I risultati emersi consentono di elaborare alcune risposte, certamente suscettibili di ulteriori approfondimenti, ai tre interrogativi da cui è scaturita questa ricerca. Innanzitutto, è possibile sostenere che, durante i mesi della quarantena, l'opportunità offerta dalle lezioni online di poter continuare l'impegno come studenti e studentesse, eludendo i rischi e i timori di interruzioni e cesure, ha certamente sortito effetti positivi. Le ricerche in campo medico e psicologico (Brooks et al., 2020) raccontano che, nelle situazioni di quarantena durante le epidemie, i giovani tra i 16 e i 24 anni sarebbero tra le categorie più predisposte a sviluppare disagio psicofisico, che può manifestarsi attraverso depressione, stress, irritabilità, ansia, insonnia, rabbia, esaurimento emotivo, difficoltà di concentrazione. Tale disagio sarebbe una risposta all'incertezza sulle proprie condizioni di salute e a una quotidianità caratterizzata da separazione dagli affetti, limitazione delle libertà personali, noia, spaesamento per la perdita delle consolidate abitudini e per la messa in dubbio delle prospettive per il futuro prossimo. Le lezioni online intervengono in modo rassicurante, offrendo una occupazione che consente di non abbandonarsi a noia e apatia e di conservare, seppure in forme surrogate ed emergenziali, la percezione di vivere la quotidianità da studente universitario a cui da mesi o anni ci si era abituati, caratterizzata da lezioni da seguire, libri e appunti da studiare, compagni e professori con cui interagire, nell'intatta prospettiva di pervenire nei tempi programmati alla laurea, veicolo di accesso a una futura carriera professionale.

Sul piano didattico, il gradimento per le lezioni online considerate come risposta a una situazione emergenziale si accompagna al riconoscimento di alcuni degli aspetti positivi a esse intrinseche, già esplicitati dalla letteratura scientifica di riferimento: la flessibilità, il risparmio economico, l'evitamento dei disagi legati agli spostamenti e al pendolarismo, la comodità del rimanere nella propria abitazione. Tra le possibili chiavi di lettura di questi esiti, riteniamo interessante riscontrare nei pregi individuati nella didattica a distanza le modalità risolutorie di atavici problemi che affliggono il sistema universitario e le organizzazioni che vi ruotano intorno. In primo luogo, è possibile menzionare l'inadeguatezza delle infrastrutture, le aule non sufficientemente capienti in cui non sempre l'acustica e le dotazioni informati- che sono ottimali, talvolta suddivise in edifici distanti tra loro, provocando in certi casi malfunzionamenti tali da indurre gli studenti a contestare l'esosità delle tasse d'iscrizione che pagano. A questi si sommano i disagi provocati dai mezzi di trasporto, in modo particolare $\mathrm{i}$ treni e gli autobus, sovente sovraffollati e in ritardo. Per dirla con altre parole, la modalità online piace a taluni perché permette di evitare le esperienze faticose di essere studenti pendolari quotidianamente alle prese con la frequentazione di strutture e trasporti inadeguati.

La mancanza di interazione fisica con i compagni e con i docenti costituisce, al contrario, un importante elemento di criticità della didattica a distanza, la cui frequente menzione suggerisce come la gran parte degli studenti riconosca l'importanza della dimensione sociale, amicale e interazionale nella propria condizione di membri della comunità accademica, anche al di là dei vantaggi che produrrebbe in termini di preparazione e apprendimento: l'essere studenti universitari non contemplerebbe solamente lo studio e il superamento degli esami, ma costituirebbe una dimensione esistenziale di più vasta portata, in cui la socialità e le relazioni assumono un ruolo di primo piano. L'ammissione da parte di alcuni di non essere a proprio agio nel porre domande e fare interventi in presenza, al cospetto di molte persone, costituisce però un elemento significativo su cui riflettere, nella direzione di una migliore gestione del clima emotivo, comunicativo e relazionale nell'aula, in modo che le spinte inibenti possano essere contrastate con maggiore efficacia. Allo stesso modo, possono sorprendere le dichiarazioni relative alla presenza di un persistente brusio e chiacchiericcio durante le lezioni, a sua volta indice di difficoltà da parte del professore nello stimolare l'attenzione e l'interesse e nel mantenere la disciplina e il rispetto.

Gli indizi raccolti dagli esiti di questa ricerca inducono a riflettere su come l'esperienza della didattica online durante la quarantena e nell'intero secondo semestre dello scorso anno accademico possa essersi rivelata una importante occasione sia per produrre una innovazione della didattica nella sua globalità, introducendo un più rodato ed efficace utilizzo delle tecnologie per sviluppare forme miste di interazioni in presenza e a distan$\mathrm{za}$, sia per ragionare e intervenire sulle magagne e sugli aspetti dell'insegnamento tradizionale maggiormente problematici e deficitari, a livello di contenuti, strumenti, strategie, organizzazione e, soprattutto, contesti.

\section{CONCLUSIONI}

Arriverà, ci auspichiamo il prima possibile, il tempo in cui verranno trovate e messe in atto contromisu- 
re al coronavirus Covid-19 e assestamenti organizzativi e logistici efficaci al punto tale da consentire un ritorno alla piena frequentazione delle lezioni nelle sedi universitarie. In quel preciso momento, la didattica online smetterà di essere una necessità legata all'emergenza, per divenire una possibilità, o meglio ancora una opportunità. Per quel che concerne i corsi elargiti dalle Scuole di Medicina e Chirurgia, caratterizzati da specificità negli obiettivi, nei contenuti, nelle esigenze strutturali e nell'utenza, i dati analizzati nel presente articolo consentono di concludere che, nella fase post-pandemica, la messa in atto di una offerta didattica mista e ibrida, che contempli e sappia valorizzare le potenzialità sia della modalità in presenza, sia di quella online, e che al contempo possa intervenire per migliorare e sopperire ai deficit di entrambe, adattandosi in modo flessibile e pragmatico alle condizioni ambientali e contestuali e agli imprevisti, potrebbe costituire la migliore delle risposte possibili a un evento che ha messo a dura prova la tenuta non solo dell'intero sistema, ma anche delle progettazioni esistenziali di milioni di studenti e studentesse, che non possono smettere di riconoscere nell'università e nella scelta di carriera intrapresa un riferimento importante nel proprio percorso di costruzione identitaria, sociale e professionale.

\section{BIBLIOGRAFIA}

Allen, I.E., \& Seaman, J. (2010). Learning on demand: Online education in the United States. Babson Survey Research Group.

Anderson, T. (2011). The theory and practice of online learning. Edmonton. AU Press.

Birnbaum, B. (2001). Foundations and practices in the use of distance education. Edwin Mellon Press.

Blumenstyk, G. (2020, March 11). Why coronavirus looks like a 'black swan' moment for higher ed.. Chronicle of Higher Education. https://www.chronicle.com/article/Why-Coronavirus-Looks-Likea/248219? cid $=$ cp 275

Bonaiuti, G. (Ed.) (2006). E-learning 2.0: il futuro dell'apprendimento in rete fra formale e informale. Erickson.

Braun, V., \& Clarke, V. (2006). Using thematic analysis in psychology. Qualitative Research in Psychology, 3 (2), 77-101. http://dx.doi. org/10.1191/1478088706qp063oa

Brooks, S.K., Webster, R.K., Smith, E.S., Woodland, L., Wessely, S., Greenberg, N., \& Rubin. G.J. (2020). The psychological impact of quarantine and how to reduce it: rapid review of the evidence. Lan- cet, 395, 912-920. https://doi.org/10.1016/S01406736(20)30460-8

Buzan, B., Waever, O., \& de Wilde, J. (1998). Security: A new framework for analysis. Lynne Reinner.

Campione, V. (Ed.) (2015). La didattica nellera digitale. Il Mulino.

Dyrud, M.A. (2000). The third wave: a position paper. Business Communication Quarterly, 63(3), 81-93. https://doi.org/10.1177/108056990006300310

Firat, M. (2016). Measuring the e-learning autonomy of distance education students. Open Praxis, 8(3), 191201. http://dx.doi.org/10.5944/openpraxis.8.3.310

Hara, N., \& Kling, R. (2000). Student distress in a webbased distance education course. Information, Communication \& Society, 3(4), 557-579.

https://doi.org/10.1080/13691180010002297

Huang, C., Wang, Y., Li, X., Ren, L., Zhao, J., Hu, Y., Zhang, L., Fan, G., Xu, J., Gu, X., Cheng, Z., Yu, T., Xia, J., Wei, Y., Wu, W., Xie, X., Yin, W., Li, H., Liu, M., \& Cao, B. (2020). Clinical features of patients infected with 2019 novel coronavirus in Wuhan, China. The Lancet, 395, 497-506. https://doi.org/10.1016/ S0140-6736(20)30183-5

Jacobs, G.M., Renandya, W.A., \& Power, M. (2016). Learner autonomy. In G. Jacobs, W.A. Renandya \& M. Power (Eds.), Simple, powerful strategies for student centered learning (pp. 19-27). Springer International Publishing.

Lai, C.C., Shih, T.P., Ko, W.C., Tang, H.J., \& Hsueh, P.R. (2020). Severe acute respiratory syndrome coronavirus 2 (SARS-CoV-2) and coronavirus disease-2019 (COVID-19): The epidemic and the challenges. International Journal of Antimicrobial Agents, 55(3), 1-9. https://doi.org/10.1016/j.ijantimicag.2020.105924

Margiotta, U. (Ed.) (1999). Pensare in rete. La formazione del multialfabeta. Bologna: CLUEB.

Matthews, D. (1999). The origins of distance education and its use in the United States. T.H.E. Journal, 27(2), 56-66.

Meyer, K.A., \& Wilson, J.L. (2011). The role of online learning in the emergency plans of flagship institutions. Online Journal of Distance Learning Administration, 14. https://www.learntechlib.org/p/52628/

Munster, V.J., Koopmans, M., van Doremalen, N., van Riel, D., \& de Wit, E. (2020). A novel coronavirus emerging in China: Key questions for impact assessment. New England Journal of Medicine, 382(8), 692694. https://doi.org/10.1056/NEJMp2000929

Murphy, M.P.A. (2019). 'The continuation of sovereign capture by other means': Biopolitical tattooing and the shared logic of the exception and securitisation. 
Critical Studies on Security», 7(1), 34-50. https://doi. org/10.1080/21624887.2018.1535210

Murphy, M.P.A. (2020). COVID-19 and emergency eLearning: Consequences of the securitization of higher education for post-pandemic pedagogy. Contemporary Security Policy, 1-14. https://doi.org/10.108 $0 / 13523260.2020 .1761749$

Persico, D., \& Midoro, V. (2013). Albori di una pedagogia digitale. In D. Persico \& V. Midoro (Eds.), Pedagogia nellera digitale (pp. 7-15). Menabò.

Ranieri, M. (2011). Le insidie dell'ovvio. Tecnologie educative e critica della retorica tecno-centrica. ETS.

Sadeghi, M. (2019). A Shift from Classroom to Distance Learning: advantages and Limitations. International Journal of Research in English Education, 4(1), 80-88. DOI: 10.29252/ijree.4.1.80

Traxler, J. (2018). Distance learning: Predictions and possibilities. Education Sciences, 8(1), 35. https://doi. org/10.3390/educsci8010035

UNESCO (2020, 13 marzo). COVID-19 educational disruption and response. https://en.unesco.org/themes/ education-emergencies/coronavirus-school-closures

Waever, O. (1995). Securitization and desecuritization. In R. Lipschutz (Ed.), On security (pp. 46-86). Columbia University Press.

World Health Organization. (2020, March 11). WHO Director-General's opening remarks at the media briefing on COVID-19. https://www.who.int/director-general/speeches/detail/who-director-general-sopening-remarks-at-the-media-briefing-on-covid19---11-march-2020 